\title{
Principais Aspectos da Polimerização do 1,3-Butadieno
}

\author{
Denise S. S. Nunes, Fernanda M. B. Coutinho \\ Instituto de Química, UERJ
}

\author{
Ivana L. Mello \\ Instituto de Macromoléculas Prof. Eloisa Mano, UFRJ
}

\begin{abstract}
Resumo: Atualmente, há uma grande demanda pelo polibutadieno com alto teor de unidades 1,4-cis, devido às suas excelentes propriedades físicas, tais como alta resistência à abrasão, baixo desenvolvimento de calor, baixa resistência ao rolamento, maior resistência à tensão, alta resistência à fadiga, baixa histerese e alta resistência à fratura. Tais características fazem com que esse elastômero seja cada vez mais utilizado na indústria pneumática. Assim, este trabalho apresenta uma breve revisão sobre a polimerização do 1,3-butadieno com diferentes sistemas catalíticos, sendo os sistemas à base de lantanídeos o principal foco desta revisão, pois esses sistemas são os mais estereoespecíficos para a polimerização 1,4cis do butadieno.
\end{abstract}

Palavras-chave: Polibutadieno alto-cis, catalisador Ziegler-Natta, neodímio.

\section{Principles Aspects of Butadiene 1,3 Polymerization}

Abstract: Nowadays, there is a great demand for polybutadiene with high contents of cis groups owing to its excellent physical properties, such as high abrasion resistance and low heat build up. These characteristics make this elastomer one of the most used in pneumatic industry. Thus, this paper presents a brief review about the 1,3-butadiene polymerization by different catalysts systems, with the systems based on lanthanides being the main focus, as they are the most stereospecific for cis-1,4 polymerization of butadiene.

Keywords: High cis-1,4 polybutadiene, Ziegler-Natta catalyst, neodymium.

\section{Introdução}

Desde muito cedo, houve um grande esforço no sentido de produzir borracha sintética. No princípio da Primeira Grande Guerra foram obtidos tipos inferiores de borracha a partir de 2,3-dimetilbutadieno na Alemanha e na antiga União Soviética. Esse fato aliado ao custo mais baixo e maior grau de pureza do 1,3-butadieno foi a mola propulsora para o desenvolvimento da pesquisa para obtenção de borrachas à base de polibutadieno ${ }^{[1-4]}$.

A pesquisa realizada no período de 1920 a 1930 levou ao desenvolvimento da copolimerização do butadieno e estireno, produzindo Buna $\mathrm{S}$ e da copolimerização de butadieno e acrilonitrila, que produziu um elastômero (NBR) com boa resistência a óleos e solventes apolares. Os resultados obtidos nessas pesquisas foram de grande importância para o desenvolvimento de novos tipos (grades) de borrachas sintéticas. Entretanto, esses novos produtos não apresentavam propriedades elásticas tão boas como as da borracha natural, proveniente da seringueira, como a Hevea Brasiliensis ${ }^{[1]}$.

Vários foram os fatores que contribuíram para a procura de alternativas de síntese de elastômeros com boas pro- priedades elásticas ${ }^{[1-6]}$. Entre esses, o preço da borracha natural, que vinha em constante crescimento desde 1902 , atingiu o seu custo mais elevado em 1910. Esse fato foi suficiente para dar início na Alemanha à pesquisa sobre borracha sintética visando o desejo de auto-suficiência nacional para atender à demanda tecnológica. $\mathrm{O}$ início da II Guerra Mundial, que provocou a suspensão do fornecimento de borracha natural para os Estados Unidos devido à invasão das áreas produtoras (Malásia) pelo Japão, levou os americanos a se organizarem para a planificação de uma indústria de borracha sintética em território nacional, que se expandiu até os dias de hoje. A partir da II Guerra Mundial, o crescimento da indústria petroquímica aumentou o fornecimento da matéria-prima para o desenvolvimento da indústria de monômeros e, paralelamente, da indústria de polímeros. Além disso, outro fator importante foi a expansão considerável da indústria automotiva nesse período e a conseqüente impossibilidade da borracha natural atender a tal demanda. Finalmente, as vantagens técnico-econômicas oferecidas pela borracha sintética em algumas aplicações.

Após a II Guerra Mundial, a química dos compostos organometálicos e a polimerização estereoespecífica, com a grande contribuição de Ziegler e Natta, abriram novas

Autor para correspondência: Fernanda M. B. Coutinho, Instituto de Química, UERJ, Av. Maracanã 524 - Pavilhão Haroldo Lisboa da Cunha, CEP: 20559900, Rio de Janeiro, RJ. E-mail: fern@uerj.br 
Tabela 1. Características dos vários isômeros do polibutadieno

\begin{tabular}{lcccc}
\hline \multicolumn{1}{c}{ Isômero } & $\begin{array}{c}\text { Característica } \\
\text { Física }\end{array}$ & Densidade & $\begin{array}{c}\mathbf{T g} \\
\left({ }^{\circ} \mathbf{C}\right)\end{array}$ & $\begin{array}{c}\mathbf{T m} \\
\left({ }^{\circ} \mathbf{C}\right)\end{array}$ \\
\hline $\begin{array}{l}\text { 1,4-cis } \\
\text { Amorfo } \\
\text { (elastômero) }\end{array}$ & $0,93-1,02$ & -110 & $2-4$ \\
$\begin{array}{l}\text { 1,4-trans } \\
\text { Cristalino }\end{array}$ & 1,01 & -110 & $143-145$ \\
$\begin{array}{l}\text { 1,2-vinila } \\
\text { 1sotático }\end{array}$ & Cristalino & 0,96 & $0-10$ & $120-128$ \\
$\begin{array}{l}\text { 1,2-vinila } \\
\text { sindiotático }\end{array}$ & Cristalino & 0,96 & $0-10$ & $154-156$ \\
$\begin{array}{l}\text { 1,2-vinila } \\
\text { atático }\end{array}$ & Amorfo & - & $0-10$ & - \\
\hline
\end{tabular}

$\overline{\mathrm{Tg}}$ = temperatura de transição vítrea; $\mathrm{Tm}=$ temperatura de fusão cristalina

Tabela 2. Características físicas e aplicações dos diferentes tipos de polibutadieno

\begin{tabular}{|c|c|}
\hline Tipos de polibutadienos & $\begin{array}{l}\text { Características físicas do } \\
\text { polímero a temperatura } \\
\text { ambiente e aplicações }\end{array}$ \\
\hline $\begin{array}{c}\text { Baixo 1,4-cis } \\
\text { (<91\% de unidades } 1,4 \text { cis }) \\
\text { Médio 1,4-cis } \\
\text { (92 a 96\% de unidades } 1,4 \text { cis }) \\
\text { Alto } 1,4-c i s \\
\text { (> 97\% de unidades } 1,4 \text { cis })\end{array}$ & $\begin{array}{l}\text { Após aditivação tem aplicação } \\
\text { semelhante à da borracha natural, } \\
\text { principalmente na fabricação de } \\
\text { pneus (com bom desempenho em } \\
\text { climas frios); em correias trans- } \\
\text { portadoras, solados para calçados, } \\
\text { isolante para cabos, fios, brinque- } \\
\text { dos, bolas de golfe. }\end{array}$ \\
\hline Alto 1,4-trans & $\begin{array}{l}\text { Utilização restrita somente para } \\
\text { reforço de pneus, pois é um termo- } \\
\text { plástico altamente cristalino e com } \\
\text { alto ponto de fusão. }\end{array}$ \\
\hline Médio 1,4-trans & $\begin{array}{l}\text { É um elastômero utilizado em } \\
\text { mistura com outras borrachas sin- } \\
\text { téticas ou com a borracha natural. } \\
\text { Aplicado em banda de rodagem de } \\
\text { pneus; toner e cintos de segurança. }\end{array}$ \\
\hline 1,2-vinila-isotático & $\begin{array}{l}\text { Polímero cristalino, sem aplicação } \\
\text { comercial significativa. }\end{array}$ \\
\hline 1,2-vinila-sindiotático & $\begin{array}{l}\text { Polímero cristalino, combina as } \\
\text { propriedades de plástico e borracha. } \\
\text { Utilizado na fabricação de fibras de } \\
\text { carbono ou grafite. }\end{array}$ \\
\hline 1,2-vinila-atático & $\begin{array}{l}\text { Elastômero amorfo, utilizado } \\
\text { principalmente em formulações } \\
\text { para pneus. }\end{array}$ \\
\hline
\end{tabular}

linhas de pesquisa e a descoberta de novas estruturas macromoleculares.

Em 1947, Morton relatou que a mistura de três sais de sódio (alil-sódio, isopropóxido de sódio e cloreto de sódio), o catalisador Alfin (nome proveniente de álcool e olefina), polimerizou butadieno, produzindo o isômero 1,4-trans do polibutadieno. Os catalisadores Alfin eram também capazes de polimerizar além do butadieno, o isopreno, o estireno e suas misturas. Entretanto, os polibutadienos produzidos por catalisadores Alfin não são usados comercialmente devido ao seu alto peso molecular, o que dificulta o seu processamento ${ }^{[7,9]}$.

No final da década de 50, o Instituto de Química Industrial do Politécnico de Milão e a Montecatini fizeram uma parceria, que incluía o desenvolvimento de catalisadores de metais de transição e compostos organometálicos para a polimerização isotática de propileno. Esses estudos foram a base para o desenvolvimento de sistemas catalíticos para a polimerização de dienos conjugados ${ }^{[6]}$.

Surgiram então as borrachas sintéticas estereorregulares, dentre elas as que mais se destacaram foram as de polisopreno e as de polibutadieno com um elevado grau de regularidade (estrutura 1,4-cis). O produto sintético tem a vantagem de ser mais puro e de qualidade mais uniforme. Em virtude do suprimento de butadieno ser mais abundante e mais barato que o do isopreno, o polibutadieno estereorregular tornou-se um dos segmentos da indústria de borracha sintética que vem sendo comercializado com maior destaque. Esse polibutadieno está sendo utilizado em pneus, pois possui excepcional resistência à abrasão e baixo desenvolvimento de calor (heat build-up) ${ }^{[1]}$.

Várias pesquisas são realizadas em todo mundo com o objetivo de atender os principais mercados de elastômeros. Estão em desenvolvimento novos sistemas catalíticos, mais ativos, para a produção de diferentes tipos dos principais elastômeros (homopolímeros, copolímeros, e mesmo terpolímeros). No campo da tecnologia de elastômeros, estão em desenvolvimento novas composições elastoméricas visando atender a crescente demanda do setor e as rigorosas exigências do mercado mundial. Projetos mais recentes estão direcionados para o desen-

Tabela 3. Principais propriedades dos elastômeros diênicos após a vulcanização

\begin{tabular}{|c|c|}
\hline Elastômero & $\begin{array}{l}\text { Propriedades após a } \\
\text { vulcanização }\end{array}$ \\
\hline $\begin{array}{l}\text { Poli-cis-isopreno natural } \\
\text { (borracha natural -NR) }\end{array}$ & $\begin{array}{l}\text { - grande elasticidade } \\
\text { - baixa histerese } \\
\text { - baixa deformação permanente }\end{array}$ \\
\hline Poliisopreno (IR) & - quase iguais às da NR \\
\hline \multirow[t]{2}{*}{ Polibutadieno alto cis (BR) } & $\begin{array}{l}\text { - após adição de carga reforçadora } \\
\text { (negro de fumo) suas propriedades } \\
\text { se situam entre as propriedades do } \\
\text { SBR e da NR - baixa histerese }\end{array}$ \\
\hline & $\begin{array}{l}\text { - maior resistência à abrasão do } \\
\text { que a NR - processabilidade } \\
\text { semelhante à da NR }\end{array}$ \\
\hline $\begin{array}{l}\text { Copolímero de butadieno- } \\
\text { estireno (SBR) }\end{array}$ & $\begin{array}{l}\text { - precisa de carga reforçadora } \\
\text { (negro de fumo) para apresentar } \\
\text { resistência mecânica satisfatória. } \\
\text { - maior histerese do que a NR } \\
\text { - menor resiliência do que a NR } \\
\text { - processabilidade semelhante à } \\
\text { NR }\end{array}$ \\
\hline $\begin{array}{l}\text { copolímero de butadieno- } \\
\text { acrilonitrila (NBR) }\end{array}$ & $\begin{array}{l}\text { - precisa de carga reforçadora } \\
\text { (negro de fumo) para apresentar } \\
\text { resistência mecânica satisfatória. } \\
\text { - alta histerese } \\
\text { - baixa resiliência } \\
\text { - processabilidade semelhante à da } \\
\text { NR }\end{array}$ \\
\hline
\end{tabular}


Tabela 4. Sistemas catalíticos à base de metais de transição (orbitais d) utilizados na obtenção de polibutadieno 1,4-cis e 1,4-trans

Polibutadieno alto 1,4-cis $(97$ a $100 \%)$

\begin{tabular}{|c|c|c|}
\hline Sistemas à base de cobalto & Sistemas à base de titânio & Sistemas à base de niquel \\
\hline $\begin{array}{l}\mathrm{AlEt}_{2} \mathrm{Cl}+\text { bis(aldeído salicílico de Co (II) } \\
\mathrm{AlR}_{2} \mathrm{Cl}+\mathrm{CoCl}_{2} \cdot \mathrm{EtOH} \\
\mathrm{LiBH}_{4}+\mathrm{CoCl}_{2} \cdot \mathrm{AlCl}_{3} \\
\mathrm{AlEt}_{2} \mathrm{Cl}+\mathrm{CoCl}_{2} \cdot 2 \text {-piridina } \\
\mathrm{AlEt}_{2} \mathrm{Cl}+\mathrm{sais} \mathrm{de} \mathrm{cobalto} \\
\left(\mathrm{AlEt}_{2} \mathrm{Cl}+\mathrm{CoCl}_{2} \cdot(\mathrm{RO})_{3} \mathrm{PO}\right) \\
\mathrm{AlEt} 2 \mathrm{Cl}+\text { dinonilnaftaleno sulfonato de } \\
\text { cobalto } \\
\mathrm{AlR}_{2} \mathrm{Cl}+\mathrm{Co}\left(\mathrm{OCOR}^{\prime}\right)_{2} \\
\mathrm{Al}_{2} \mathrm{Et}_{3} \mathrm{Cl}_{3}+\mathrm{Co}\left(\mathrm{OCOR}^{\prime}\right)_{2} \\
\mathrm{Aloxano}+\mathrm{Co}(\text { acac })_{3} \\
\text { (comercialmente importantes) }\end{array}$ & $\begin{array}{l}\mathrm{AlEt}_{3}+\mathrm{TiCl}_{4}+\mathrm{I}_{2} \\
\mathrm{AlEt}_{3}+\mathrm{TiCl}_{4}+\mathrm{AlI}_{3} \\
\mathrm{AlEt}_{3}+\mathrm{TiCl}_{4}+\mathrm{AlEt}_{2} \mathrm{I} \\
\text { Podem ser adicionados doadores de } \\
\text { elétrons em pequenas proporções (éteres, } \\
\text { tioéteres e aminas terciárias) aos } \\
\text { sistemas mencionados acima. }\end{array}$ & $\begin{array}{l}\mathrm{AlEt}_{3}+\text { sais de } \mathrm{Ni} \\
\mathrm{AlEt}_{3}+\text { sais de } \mathrm{Ni}+\mathrm{BF}_{3} \text { ou outros } \\
\text { fluoretos metálicos } \\
\mathrm{AlEt}_{3} / \mathrm{LiBu}+\text { isopropil salicilato de } \\
\text { níquel } \\
\mathrm{AlEt}_{3}+\mathrm{Ni} \text { (carboxilato) }+\mathrm{HF} \\
\mathrm{AlEt}_{3}+\mathrm{Ni} \text { (carboxilato) }+\mathrm{BF}_{3} \cdot \mathrm{OEt}_{2} \\
\text { (comercialmente importantes) }\end{array}$ \\
\hline \multicolumn{3}{|c|}{ Polibutadieno médio 1,4-cis (92 a 96\%) } \\
\hline Sistemas à base de cobalto & Sistemas à base de titânio & Sistemas à base de níquel \\
\hline $\begin{array}{l}\mathrm{LiAlH}_{4}+\text { complexo (octanoato de } \mathrm{Co}- \\
\mathrm{AlCl}_{3} \\
\mathrm{MAO}+\mathrm{Co}(\mathrm{acac})_{3}\end{array}$ & $\begin{array}{l}\mathrm{AlR}_{3}+\mathrm{TiI}_{4}(\mathrm{Al} / \mathrm{Ti}=3-6)^{*} \\
\mathrm{AlR}_{3}+\mathrm{AlEt}_{2} \mathrm{I}+\mathrm{TiCl}_{4} \\
\mathrm{Al}\left(\mathrm{i}-\mathrm{C}_{4} \mathrm{H}_{9}\right)_{3}+\mathrm{TiCl}_{4}+\mathrm{I}_{2} \\
\text { Hidretos de } \mathrm{Al}+\mathrm{AlI}_{3}+\mathrm{TiCl}_{4} \\
\mathrm{LiAlH}_{4}+\mathrm{TiCl}_{2} \mathrm{I}_{2} \\
\mathrm{LiAlH}_{4}+\mathrm{TiCl}_{4}+\mathrm{I}_{2}\end{array}$ & $\mathrm{AlEt}_{3}+\mathrm{Ni}($ naftenato $)+\mathrm{BF}_{3}($ eterato $)$ \\
\hline \multicolumn{3}{|c|}{ Polibutadieno baixo 1,4-cis (57 a $91 \%$ ) } \\
\hline Sistemas à base de cobalto & Sistemas à base de titânio & Sistemas à base de níquel \\
\hline $\mathrm{AlEt}_{2} \mathrm{Cl}+\mathrm{Co}(\mathrm{CO})_{4}$ & $\mathrm{AlR}_{3}+\mathrm{TiCl}_{4}(\mathrm{Al} / \mathrm{Ti}=1,2)^{*}$ & $\mathrm{AlEt}_{3}+\mathrm{Ni}$ (naftenato $)+\mathrm{BF}_{3}$ (eterato $)$ \\
\hline \multicolumn{3}{|c|}{ Polibutadieno 1,4-trans } \\
\hline Sistemas à base de cobalto & Sistemas à base de titânio & Sistemas à base de vanádio \\
\hline $\begin{array}{l}\mathrm{AlEt}_{2} \mathrm{Cl}+\mathrm{Co}(\mathrm{acac})_{2} \\
\mathrm{AlEt}_{3}+\mathrm{Co}(\mathrm{acac})_{2} /(\mathrm{THF} \text { como solvente }) \\
\mathrm{AlEt}_{2} \mathrm{Cl}+\mathrm{CoCl}_{2}+\mathrm{CS}_{2}+\mathrm{RC}_{6} \mathrm{H}_{4} \mathrm{OH}\end{array}$ & $\begin{array}{l}\mathrm{AlR}_{3}+\mathrm{TiCl}_{4}(\mathrm{Al} / \mathrm{Ti}=1,0) \\
\mathrm{AlEt}_{3}+\alpha-\mathrm{TiCl}_{3} \\
\mathrm{AlEt}_{3}+\mathrm{TiCl}_{3}+\mathrm{AlI}_{3} \\
\mathrm{Al}\left(\mathrm{i}-\mathrm{C}_{4} \mathrm{H}_{9}\right)_{3}+\mathrm{TiCl}_{4} \\
\mathrm{CdEt}_{2}+\mathrm{TiCl}_{4}(\mathrm{Cd} / \mathrm{Ti}=5,0)^{*} \\
\mathrm{CdR}_{2} \mathrm{Cl}+\mathrm{Ti}\left(\mathrm{NEt}_{2}\right)_{4}\end{array}$ & $\begin{array}{l}\mathrm{AlEt}_{2} \mathrm{Cl}+\mathrm{V}(\mathrm{acac})_{3} \\
\mathrm{AlEt}_{2} \mathrm{Cl}+\mathrm{VCl}_{3} \cdot 3 \mathrm{THF} \\
\mathrm{AlEt}_{3}+\mathrm{VCl}_{3} \\
\mathrm{AlEt}_{2} \mathrm{Cl}+\mathrm{VOCl}_{3} \\
\mathrm{AlEt}_{2} \mathrm{Cl}+\mathrm{VCl}_{4}\end{array}$ \\
\hline
\end{tabular}

$\mathrm{R}=$ alquila, acac $=$ acetilacetonato $; \mathrm{THF}=$ tetra-hidrofurano; $\mathrm{TMEDA}=\mathrm{N}, \mathrm{N}, \mathrm{N}$ ', N' - tetrametiletilenodiamina

*Todas as relações mencionadas na tabela são relações molares.

volvimento dos "pneus verdes", pneus mais leves, com mais baixa resistência ao rolamento. Esses desenvolvimentos têm como objetivos principais o aumento da vida útil do pneu e a economia de combustível, com o conseqüente decréscimo da emissão de gases poluentes na atmosfera ${ }^{[2-9]}$.

\section{Estruturas, características físicas e aplicações do polibutadieno e seus copolímeros}

Cinco tipos de estruturas isoméricas podem ser formadas pela polimerização do 1,3-butadieno:1,2-vinila-isotático, 1,2vinila-sindiotático, 1,2-vinila-atático, 1,4-cis e 1,4-trans. O tipo de estrutura isomérica determina as características físicas, as propriedades e as conseqüentes aplicações do polibutadieno (Tabelas 1 e 2) (2-9]. $^{[2}$.

O mais importante dos isômeros do polibutadieno é o 1,4cis que é um elastômero a temperatura ambiente, e após aditivação tem propriedades semelhantes às da borracha natu- ral. Esse polibutadieno pode ser ainda classificado em baixo 1,4-cis, médio 1,4-cis e alto 1,4-cis, em função do teor de unidades repetitivas 1,4-cis (Tabela 2 ). As suas excelentes propriedades elastoméricas fizeram com que se tornasse um material de grande importância econômica e estratégica. Assim, há um grande número de trabalhos acadêmicos e de patentes que descrevem várias rotas para a síntese de catalisadores ZieglerNatta à base de diferentes metais de transição para a produção de 1,4-cis-polibutadieno. Há ainda um grande número de trabalhos e patentes sobre os copolímeros butadiênicos visando o desenvolvimento de materiais com melhores propriedades físicas e mecânicas (Tabela 3).

Polimerização de 1,3-dienos com iniciadores de radicais livres, catiônicos e aniônicos

A literatura ${ }^{[2,4,7-10]}$ descreve o comportamento de diferentes tipos de iniciadores (catalisadores) nas reações de 
Tabela 5. Sistemas catalíticos para a obtenção de polibutadienos 1,2-vinila-isotático, 1,2-vinila-sindiotático e 1,2-vinila-atático

\begin{tabular}{|c|c|c|}
\hline \multicolumn{3}{|c|}{ Polibutadieno alto 1,2-Vinila-Isotático } \\
\hline Sistemas à base de titânio & \multicolumn{2}{|c|}{ Sistemas à base de Cromo } \\
\hline $\mathrm{AlEt}_{3} \mathrm{Cl}+\mathrm{Ti}\left(\mathrm{O}-\mathrm{nBu}_{4}\right)$ & $\begin{array}{l}\mathrm{AlEt}_{3}+\mathrm{Cr}\left(\mathrm{CNC}_{6} \mathrm{H}_{5}\right)_{6} \\
\mathrm{AlEt}_{3}+\mathrm{Cr}(\mathrm{acac})_{3} \\
\mathrm{AlEt}_{3}+\mathrm{Cr}(\mathrm{CO})_{6} \\
\mathrm{AlEt}_{3}+\text { derivados de } \mathrm{Cr}\end{array}$ & $(\mathrm{Al} / \mathrm{Cr}=5-10)^{*}$ \\
\hline \multicolumn{3}{|c|}{ Polibutadieno 1,2-Vinila-Sindiotático } \\
\hline Sistemas à base de Cobalto & Sistemas à base de Titânio & Sistemas à base de Cromo \\
\hline$(\mathrm{AlEtCl})_{2} \mathrm{SO}_{4}+\mathrm{Co}(\mathrm{SCN})_{2}$ & $\begin{array}{l}\mathrm{AlEt}_{3}+\text { acetilacetonato de } \mathrm{Ti} \\
\mathrm{AlEt}_{3}+\text { alcoolatos de } \mathrm{Ti}^{2}\end{array}$ & $\begin{array}{l}\mathrm{AlEt}_{3}+\mathrm{Cr}(\mathrm{acac})_{3} \\
\mathrm{AlEt}_{3}+\text { alcoolato de } \mathrm{Cr} \\
\mathrm{AlEt}_{3}+\mathrm{Cr}\left(\mathrm{CNC}_{6} \mathrm{H}_{5}\right)_{6} \\
\mathrm{AlEt}_{3}+\mathrm{Cr}(\mathrm{acac})_{3} \\
\mathrm{AlEt}_{3}+\mathrm{Cr}(\mathrm{CO})_{6} \\
\mathrm{AlEt}_{3}+\text { derivados de } \mathrm{Cr} \\
(\mathrm{Al} / \mathrm{Cr}=2,5-3,0)^{*} \\
\mathrm{MAO}+\mathrm{CoCl}_{2}+\mathrm{DMPE}\end{array}$ \\
\hline Sistemas à base de Vanádio & \multicolumn{2}{|c|}{ Sistemas à base de Molibidênio } \\
\hline $\begin{array}{l}\mathrm{AlEt}_{3}+\text { acetilacetonatos de V } \\
\mathrm{AlEt}_{3}+\text { alcoolatos de V }\end{array}$ & & $\begin{array}{l}\mathrm{AlEt}_{3}+\mathrm{MoCl}_{5} \\
\mathrm{AlEt}_{3}+\mathrm{MoO}_{2}(\mathrm{OR})_{2} \\
\mathrm{AlEt}_{3}+\mathrm{MoO}_{2}(\mathrm{OR})_{2} \\
\mathrm{AlEt}_{3}+\mathrm{Mo}(\mathrm{acac})_{3}\end{array}$ \\
\hline
\end{tabular}

Polibutadieno 1,2-Vinil-Atático

Obtido por polimerização aniônica (hidrocarboneto /organolítio/base de Lewis)

$[\mathrm{Li}(\mathrm{n}-\mathrm{Bu})+\mathrm{THF}] /$ hexano

$[\mathrm{Li}(\mathrm{n}-\mathrm{Bu})+\mathrm{TMEDA}] /$ hexano

Sistemas catalíticos Ziegler-Natta produzem rendimentos baixos

$\mathrm{R}=$ alquila, acac $=$ acetilacetonato; $\mathrm{THF}=$ tetra-hidrofurano; $\mathrm{MAO}=$ metilaluminoxano, TMEDA $=\mathrm{N}, \mathrm{N}, \mathrm{N}, \mathrm{N}^{\prime}, \mathrm{N}^{\prime}-$ tetrametiletilenodiamina; DMPE $=$ 1,2- bis(dimetilfosfino)etano

*Todas as relações mencionadas na tabela são relações molares.

polimerização de 1,3-dienos. Podem ser empregados iniciadores de radicais livres, catiônicos, aniônicos ou de coordenação (Tabelas 4 e 5).

A preparação do polibutadieno via radicais livres pode ser realizada em massa, em solução ou em emulsão. Em massa, são obtidas frações de baixo peso molecular (solúveis) e de peso molecular relativamente elevado (insolúvel), resultando em uma borracha de baixa qualidade e com teor de gel relativamente alto. Polibutadieno com pesos moleculares elevados é conseguido em sistema em emulsão, que apresenta grande tendência para a formação de ligações cruzadas ${ }^{[10]}$.

A polimerização radicalar de 1,3-dieno envolve a formação de um radical livre deslocalizado entre os carbonos 2 e 4 da unidade repetitiva terminal (Figura 1). A predominância da propagação 1,4 sobre a 1,2 é uma conseqüência do baixo grau de impedimento estérico no carbono-4 em relação ao carbono-2. Este tipo de comportamento é mais acentuado na polimerização do isopreno.

A preferência pela propagação 1,4-trans sobre a 1,4-cis, em sistemas via radicais livres, é conseqüência da conformação do monômero. 1,3-Dienos existem predominantemente na conformação s-trans em oposição à conformação s-cis. A

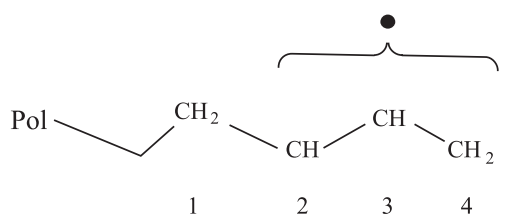

Figura 1. Estrutura do radical livre deslocalizado

formação dessas estruturas isoméricas depende da temperatura de polimerização, embora a formação da estrutura 1,4-trans seja favorecida em relação à 1,4-cis, uma vez que a conformação s-trans é mais estável que a s-cis.

A adição de um dieno ao centro de propagação ocorre com retenção da conformação do monômero, resultando em um polímero com unidades repetitivas predominantemente 1,4-trans. Esse comportamento é observado na polimerização do 1,3 -butadieno ${ }^{[10]}$.

A polimerização aniônica de 1,3-dienos resulta em polímeros com diferentes estruturas, dependendo do tipo de contra-íon e do centro de propagação. No caso de íons livres solvatados, a polimerização 1,2 é favorecida em relação à 1,4. O centro aniônico no carbono-2 não é deslocalizado, como no caso da polimerização via radicais livres. Pode-se 
Tabela 6. Sistemas catalíticos à base de metais de transição da série dos lantanídeos e actinídeos para a obtenção de polibutadieno alto-cis*

\begin{tabular}{|c|c|c|}
\hline Sistemas à base de Cério & Sistemas à base de Urânio & Sistemas à base de Neodímio \\
\hline $\begin{array}{l}\left(\mathrm{AlEt}_{3}+\mathrm{AlEtCl}_{2}\right)+\text { octanoato de } \mathrm{Ce} \\
\left(\mathrm{AlEt}_{3}+\mathrm{AlEt}_{2} \mathrm{Cl}\right)+\text { octanoato de } \mathrm{Ce}\end{array}$ & 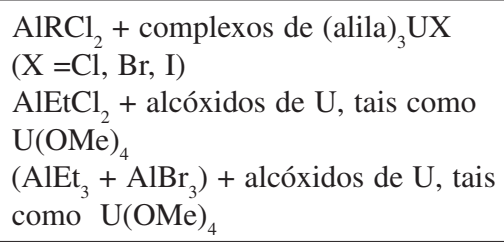 & $\begin{array}{l}\mathrm{AlEt}_{2} \mathrm{Cl}+\mathrm{Nd}(\text { carboxilato })+\mathrm{Al}\left(\mathrm{i}-\mathrm{C}_{4} \mathrm{H}_{9}\right)_{3} \\
\mathrm{Al}\left(\mathrm{i}-\mathrm{C}_{4} \mathrm{H}_{9}\right)_{3}+\mathrm{NdCl}_{3}+\text { doador de } \\
\text { elétrons (THF, álcool) }\end{array}$ \\
\hline Características & Características & Características \\
\hline $\begin{array}{l}\text { - polímero isento de gel; } \\
\text { - atividade catalítica inferior aos sistemas } \\
\text { catalíticos à base de Co e Ni, porém } \\
\text { superiores aos de } \mathrm{Ti}\end{array}$ & $\begin{array}{l}\text { - rendimentos elevados são obtidos } \\
\text { somente quando o catalisador é } \\
\text { envelhecido; } \\
\text { - polímeros com alto peso molecular; } \\
\text { - polímeros com polidispersão larga }\end{array}$ & $\begin{array}{l}\text { - polímero isento de gel; } \\
\text { - peso molecular do polímero é } \\
\text { dependente do tempo de polimerização } \\
\text { (diferença marcante entre estes } \\
\text { catalisadores e os de Ni ou Co); } \\
\text { - polímeros com polidispersão larga } \\
\text { (Mn/Mn=5 a 8) } \\
\text { - conversão total do monômero }\end{array}$ \\
\hline
\end{tabular}

* Teor de unidades 1,4-cis na faixa de 97 a $100 \%$.

observar este comportamento tanto para o isopreno como para o butadieno. Entre os iniciadores aniônicos, quando o contra-íon é o lítio, há uma tendência para a polimerização 1,4 , porém são obtidos rendimentos baixos e sempre com mistura de isômeros (1,4-cis/1,4-trans/1,2-vinila na proporção de $35 / 55 / 10)$. A natureza do metal alcalino e o tipo de solvente empregado regulam a microestrutura do polibutadieno ${ }^{[2,10]}$.

A polimerização catiônica de 1,3-dienos resulta em polímeros de pesos moleculares relativamente baixos, contendo estruturas ciclizadas.

A característica comum dos iniciadores de radicais livres, aniônicos e catiônicos é que uma vez iniciada a reação de polimerização, a adição de cada unidade monomérica se passa pelo extremo oposto ao fragmento do iniciador ligado à cadeia polimérica. O iniciador não participa diretamente da etapa de propagação. Ao contrário, nas reações de polimerização com catalisadores Ziegler-Natta, polimerização por coordenação, a inserção de novas unidades monoméricas se dá pelo rompimento da ligação do fragmento do catalisador com a última unidade monomérica inserida. A característica principal desses sistemas é a participação do catalisador na etapa de propagação, possibilitando assim o controle estereoquímico do polímero.

\section{Polimerização estereoespecífica de 1,3-dienos}

Os sistemas catalíticos Ziegler-Natta, utilizados na polimerização estereoespecífica de 1,3-dienos, são constituídos de um modo geral, por um composto organometálico e um composto de metal de transição. Dentre os organometálicos se destacam os alquilalumínios. Em geral, organometálicos de metais do Grupo IA da Tabela Periódica favorecem a adição 1,2 , enquanto as estruturas 1,4 são favorecidas por compostos de metais dos Grupos IIA e IIIA. Dentre os compostos de metais de transição podem ser citados os sais de Ti, Co, Ni e lantanídeos, como por exemplo, halogenetos, alcoolatos, acetilacetonatos e sais de ácidos orgânicos ${ }^{[3,4,6]}$.
Tabela 7. Sistemas catalíticos à base de lantanídeos para a produção de polibutadieno alto-cis

\begin{tabular}{|c|c|}
\hline Grupos & Sistemas Catalíticos \\
\hline I & $\begin{array}{l}\text { - Organoalumínio/haleto orgânico ou } \\
\text { complexo do haleto ( } \mathrm{LnX}_{3} .3 \mathrm{~L} \text {, onde: } \mathrm{L} \text { é um } \\
\text { ligante orgânico doador de elétrons, tal } \\
\text { como, o ésteres do ácido o-fosfórico, } \\
\text { sulfóxidos, álcoois e éteres cíclicos e Ln é o } \\
\text { composto de lantanídeo). }\end{array}$ \\
\hline II & $\begin{array}{l}\text { - Organoalumínio /carboxilatos de lantanídeo } \\
\text { [Ln(OCOR) } \text { (naftenatos, octanoato ou } \\
\text { estearatos), possuem boa solubilidade em } \\
\text { hidrocarbonetos e são os mais usados]. } \\
\text { - Organoalumínio/organofosfatos de } \\
\text { lantanídeo - Ln(OPOR) } \\
\text { - Organoalumínio /alcoolatos de lantanídeo } \\
(\text { Ln(OR) })_{3} \text { ) } \\
\text { - Esses sistemas incluem ainda componentes } \\
\text { contendo halogênio (haletos de } \\
\text { organoalumínio, haletos de organotitânio, } \\
\text { haletos orgânicos e halogênios em sua forma } \\
\text { elementar), que têm a função de halogenar o } \\
\text { lantanídeo. } \\
\text { - Esses sistemas apresentam a mesma } \\
\text { estereoespecificidade dos sistemas baseados } \\
\text { em haletos de lantanídeos. }\end{array}$ \\
\hline III & $\begin{array}{l}\text { - Organoalumínio/haletos de lantanídeo ou } \\
\text { um complexo do haleto orgânico com } \\
\text { compostos doadores de elétrons, contendo } \\
\text { um radical orgânico (R') (Ex: } \\
\left.\text { AlR }_{3} / \mathrm{R}^{\prime} \operatorname{L} n \mathrm{X}_{2} \cdot \mathrm{nTHF}\right)\end{array}$ \\
\hline
\end{tabular}

Nem todas as combinações de organometálicos e sais de metais de transição são efetivas, muitas são ativas apenas para certos monômeros ou em determinadas condições. A obtenção de polidienos estereorregulares depende de fatores que atuam tanto na síntese do sistema catalítico (razão entre os componentes do sistema catalítico, presença ou ausência de 
pré-polímero, doadores de elétrons, natureza do solvente, ordem de adição dos componentes, adição de dienos, etc) quanto na etapa de polimerização (temperatura, tempo, envelhecimento ou não do sistema catalítico, etc) ${ }^{[21-52]}$.

\section{Polimerização estereoespecífica de 1,3-dienos com sistemas catalíticos à base de metal de transição da série dos lantanídeos e actinídeos}

Polibutadienos com alto teor de unidades 1,4-cis (98\%) podem ser obtidos a partir de metais da série dos lantanídeos e actinídeos, dentre os mais ativos estão os derivados de cério, neodímio e urânio. A grande vantagem desses sistemas é o baixo teor de unidades vinílicas produzidas $(<1 \%)$ que, sendo pontos de imperfeição da cadeia polimérica, interferem na etapa de cristalização do polibutadieno (Tabela 6) ${ }^{[2,4,7-20]}$.

Os sistemas catalíticos à base de lantanídeos podem ser divididos em três grupos distintos de acordo com a sua composição e estereoespecificidade (Tabela 7) ${ }^{[21-38]}$.

Há poucas informações na literatura ${ }^{[53,54]}$ sobre a influência da presença de dienos conjugados na síntese de catalisadores à base de neodímio. Entre os dienos conjugados utilizados estão o 2-metil-butadieno (isopreno) e o 1,3-butadieno.

Quirk ${ }^{[53]}$ estudou o sistema catalítico ternário constituído de versatato de neodímio, tetracloreto de silício e hidreto de diisobutilalumínio (ou trietilalumínio) na polimerização estereoespecífica de 1,3-butadieno. O autor preparou o sistema catalítico em ausência e em presença de isopreno e deixou-o envelhecer por $72 \mathrm{~h}$ a $20^{\circ} \mathrm{C}$. Contudo, não foram observadas diferenças significativas quando o catalisador foi preparado em ausência ou em presença de isopreno em relação ao teor de unidades 1,4-cis dos polibutadienos (97\%).

A literatura ${ }^{[54,55]}$ descreve um processo de polimerização de dienos conjugados com catalisadores à base de versatato de neodímio $(\mathrm{Nd})$, hidreto de diisobutilalumínio $(\mathrm{Al})$, butadieno (M) e tetracloreto de silício $(\mathrm{Cl})$, na seguinte relação molar $\mathrm{Al}: \mathrm{Nd}: \mathrm{M}: \mathrm{Cl}=15: 1: 35: 3$. Os catalisadores foram envelhecidos por diferentes períodos ( $5 \mathrm{~min}, 24 \mathrm{~h}$ e 6 dias) a $20^{\circ} \mathrm{C}$. Os polímeros produzidos com catalisadores preparados em presença de butadieno apresentaram distribuição de peso molecular mais estreita (na faixa de 1,8 a 3,0) do que aqueles preparados em ausência de butadieno (na faixa de 3,1 a 7,8). Observou-se a formação de um percentual mais baixo da fração de peso molecular elevado, quando foram utilizados catalisadores preparados em presença de 1,3-butadieno. Em todos os casos, o teor de unidades 1,4-cis do polibutadieno foi de $97 \%$, e esse teor aumentou para $98 \%$ quando os catalisadores foram envelhecidos por períodos mais longos $(>24 \mathrm{~h})$.

Nunes e colaboradores ${ }^{[56]}$ estudaram a influência da adição de dienos conjugados (isopreno e 1,3-butadieno) no sistema catalítico à base de neodímio sobre a microestrutura, o peso molecular e a distribuição de peso molecular do polibutadieno e o rendimento da polimerização. Os autores observaram que os catalisadores preparados em presença de dienos mostraramse mais ativos do que aqueles preparados em ausência de dienos. Além disso, a polidispersão (Figura 2) e o teor de unidades 1,4-cis (Figura 3) do polibutadieno não variaram com a pre-

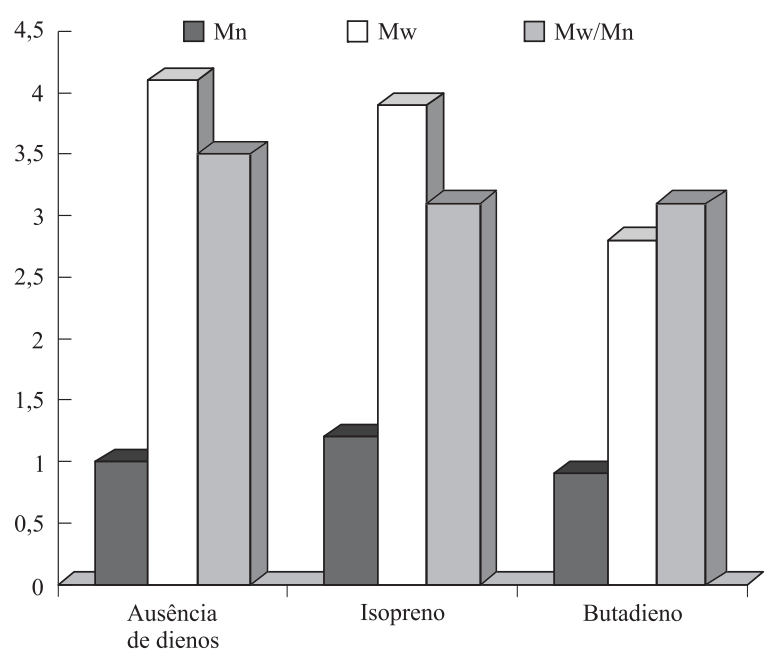

Figura 2. Influência de dienos conjugados sobre o peso molecular (Mn peso molecular numérico médio, $\mathrm{Mw}$ - peso molecular ponderal médio) e a polidispersão $(\mathrm{Mw} / \mathrm{Mn})$

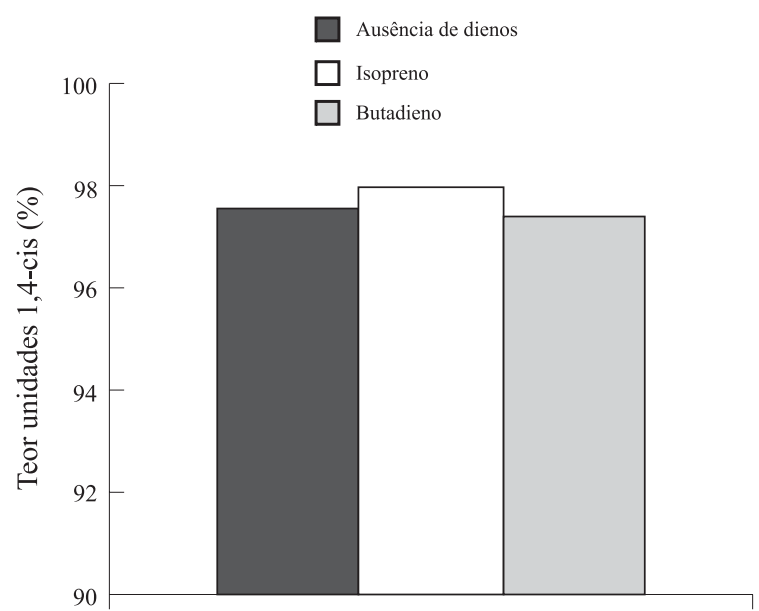

Figura 3. Influência de dienos conjugados sobre o teor de unidades 1,4-cis

sença dos dienos, enquanto que o peso molecular (Figura 2) apresentou uma tendência para diminuir.

\section{Aspectos mecanísticos}

A polimerização de 1,3-butadieno com catalisadores baseados em metais de transição é uma reação que ocorre através de um mecanismo de inserção do monômero na ligação entre o carbono (da cadeia polimérica em crescimento) e o metal de transição (do complexo catalítico).

Nos sistemas obtidos a partir de compostos de alquilalumínio e metal de transição, a primeira ligação carbono-metal é produzida na reação de formação do catalisador, ou seja, por meio da alquilação do metal de transição pelo alquilalumínio.

Para os sistemas à base de lantanídeos, a suposição de que o componente organoalumínio esteja envolvido no centro ativo baseia-se na observação de que a natureza do organoalumínio afeta, além da atividade do sistema catalítico, sua reatividade (constante de propagação da cadeia, kp), estereoespecificidade e a microestrutura do polibutadieno. Com base nessas observações o centro ativo foi considerado parte de um complexo constituído por um derivado 


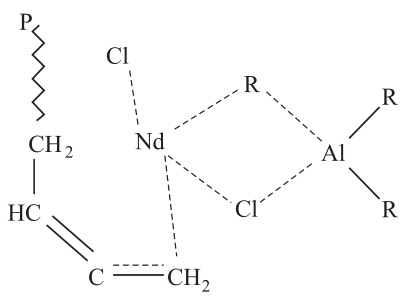

Figura 4. Estrutura do centro ativo

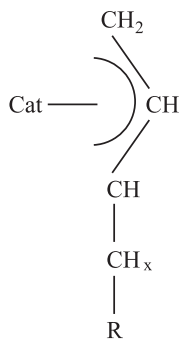

Figura 5. Representação do grupo $\eta^{3}$-butenila

halogenado do lantanídeo com outros ligantes. Essa suposição foi reforçada pelo fato de a constante de propagação de cadeia, $\mathrm{kp}$, ser dependente da natureza do halogênio. A Figura 4 mostra a estrutura proposta para o centro ativo já na etapa de propagação ${ }^{[2,3]}$.

\section{Iniciação}

A inserção da primeira molécula de monômero resulta na formação de um grupo $\eta^{3}$-butenila (Figura 5).

A ligação $\eta^{3}$-butenila entre a cadeia em crescimento e o metal de transição pode existir sob duas formas isoméricas, anti e sin, em equilíbrio (Figura 6) ${ }^{[21,57]}$.

A natureza alílica da ligação entre o metal de transição do catalisador e a última unidade monomérica inserida foi comprovada utilizando-se derivados alílicos de metais de transição como catalisador, em particular os de níquel. Esses complexos alílicos produziram somente oligômeros na polimerização de monoalquenos e sua eficiência na polimerização de 1,3-dienos foi atribuída à maior estabilidade da ligação Ni-radical alila em relação à ligação s Ni-C. Essas observações foram comprovadas por ressonância magnética nuclear (NMR).

Informações mais detalhadas, sobre a inserção de 1,3-dieno entre a ligação metal de transição e o radical alila foram obtidas por análise de ${ }^{1} \mathrm{H}$ NMR da reação entre butadieno e
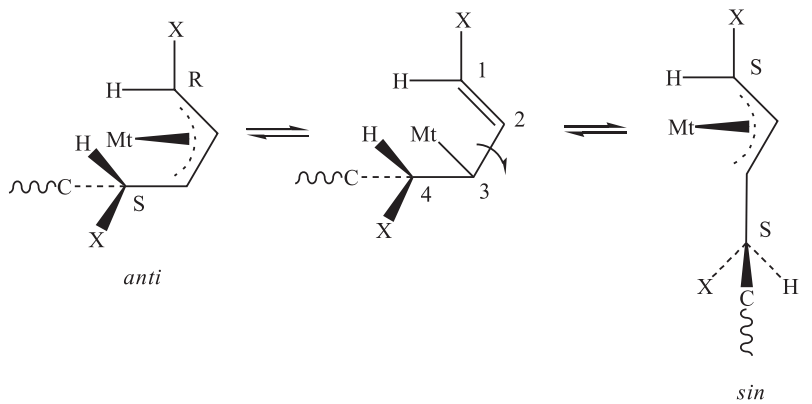

Figura 6. Equilíbrio das formas isoméricas anti e sin do grupo $\eta^{3}$-butenila
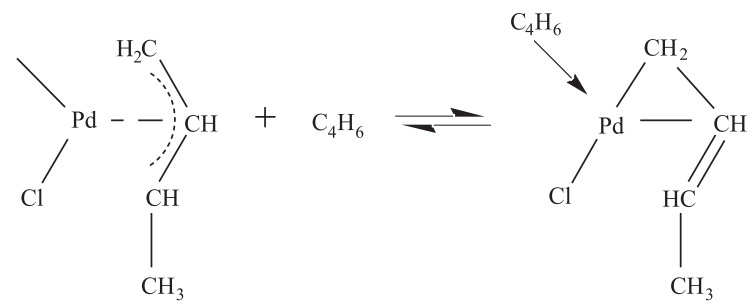

Figura 7. Equilíbrio entre as espécies $\eta^{3}$-alila e $\eta^{1}$-alila

$\left[\left(\eta^{3}-\mathrm{C}_{4} \mathrm{H}_{7}\right) \mathrm{PdCl}\right]_{2}$. Neste caso, a inserção do butadieno foi lenta. Isso permitiu observar todos os passos da reação por NMR. A primeira etapa foi a formação da espécie $\eta^{1}$-alila, resultado da coordenação de uma ou mais moléculas de monômero com o metal de transição do catalisador. Um rápido equilíbrio foi estabelecido entre as espécies $\eta^{1}$-alila e $\eta^{3}$-alila como resultado da coordenação de um doador de elétrons (monômero) com o complexo metal de transição-radical alila (Figura 7) ${ }^{[58]}$.

\section{Propagação}

A etapa de propagação consiste na continuação do processo de inserção de novas moléculas de monômero, onde ocorre o deslocamento da espécie $\eta^{1}$-alila pelo monômero. Nesse caso a formação da espécie $\eta^{1}$-alila é rápida, enquanto que o processo de inserção é mais lento.

A formação das espécies $\eta^{1}$-alila não é observada quando se utiliza derivados alílicos de níquel, pois nesse caso, a etapa de inserção é muito rápida para que essa espécie $\eta^{1}$-alila possa ser observada.

A propagação da cadeia polimérica ocorre por inserção de moléculas de monômero na ligação $\eta^{3}$-butenila existente entre o metal de transição e a última unidade monomérica inserida. Uma nova ligação $\eta^{3}$-butenila é formada após cada inserção de monômero.

A quimiosseletividade, ou seja, a formação de unidades 1,2 versus unidades 1,4 , depende do carbono do grupo $\eta^{3}$-butenila $\left(\mathrm{C}_{1}\right.$ ou $\left.\mathrm{C}_{3}\right)$ no qual ocorrerá a inserção da nova molécula de monômero. Se a inserção ocorrer em $\mathrm{C}_{1}$, resultarão unidades 1,4-cis ou 1,4-trans dependendo se o grupo

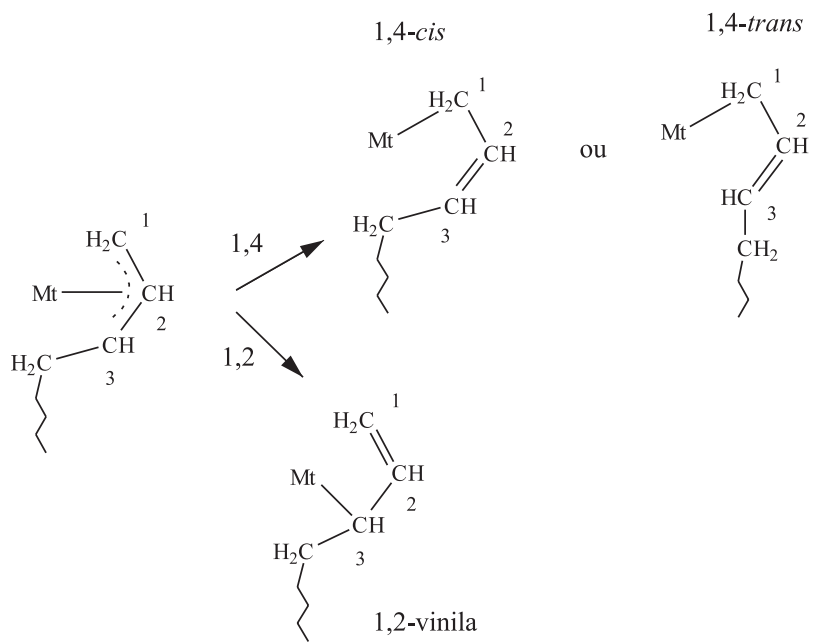

Figura 8. Quimiosseletividade da polimerização $(\mathrm{Mt}=$ Metal de transição) 
butenila for anti ou sin, respectivamente, enquanto que a inserção no $\mathrm{C}_{3}$ resultará em unidades 1,2-vinílicas, independente da forma sin ou anti do grupo butenila (Figura 8).

Os fatores que determinam em que carbono irá ocorrer a inserção são, a presença de substituintes em $\mathrm{C}_{1} \mathrm{e} e \mathrm{e} \mathrm{C}_{3}$, que poderá fazer com que um carbono seja mais reativo que o outro, o modo de coordenação do monômero e as características estruturais do complexo catalítico.

A estrutura do grupo $\eta^{3}$-butenila depende do modo de coordenação do monômero (Figura 8). Um grupo butenila anti é formado quando a coordenação do monômero é $\eta^{4}$ cis, enquanto que um grupo butenila sin pode derivar, em princípio, da coordenação $\eta^{2}$-trans ou $\eta^{4}$-trans do monômero. Contudo, também pode originar-se da isomerização anti-sin do grupo alílico. Sendo assim, os 1,3-dienos podem se coordenar com o metal de transição por meio de uma única ligação dupla formando um ligante $\eta^{2}$-trans, ou com ambas as ligações duplas formando um ligante $\mathrm{h}^{4}$-cis ou $\eta^{4}$-trans .

Sabe-se que as formas anti e sin estão em equilíbrio. Em ausência de um grupo volumoso no $\mathrm{C}_{2}$ do grupo butenila, o equilíbrio à temperatura ambiente é deslocado em direção à forma sin, que é mais estável termodinamicamente ${ }^{[21,22,58]}$.

A formação de unidades 1,4-trans versus 1,4-cis pode ser controlada pela velocidade relativa do processo de inserção da nova molécula de monômero no grupo alílico e da velocidade de isomerização anti-sin.

Se o processo de inserção for mais rápido que o processo de isomerização, resultará na formação de unidades 1,4-cis. Caso contrário, ocorrerá a formação de unidades 1,4-trans, como mostrado na Figura $9^{[23]}$.

As unidades 1,4-trans podem ser formadas via $\mathbf{a}_{1}$ ou $\mathbf{a}_{2} \rightarrow$ $a^{\prime} \rightarrow a^{\prime \prime}$ ou via $b \rightarrow b^{\prime} \rightarrow a^{\prime} \rightarrow a^{\prime \prime}$, e as unidades 1,4-cis são formadas via $\mathbf{b} \rightarrow \mathbf{b}^{\prime} \rightarrow \mathbf{b}^{\prime \prime}$ (Figura 9).

Uma mudança na estereoespecificidade é observada quando doadores de elétrons são adicionados ao sistema catalítico, que é altamente estereoespecífico para a polimerização cis de butadieno. Essa mudança na estereoespecificidade é atribuída ao fato de o doador de elétrons ocupar um sítio de coordena-

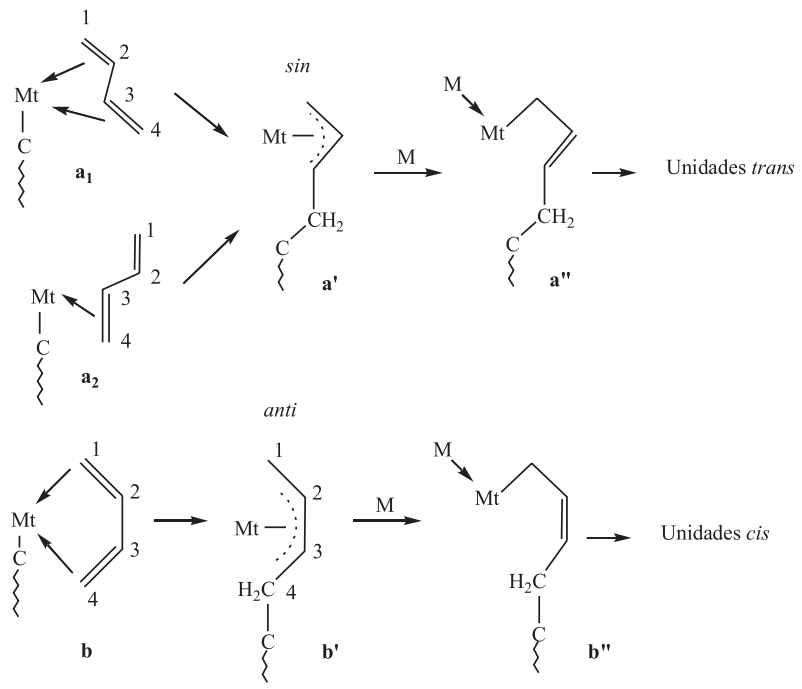

Figura 9. Mecanismo de formação das unidades 1,4-cis e 1,4-trans ção, ou seja, ocupar uma das vacâncias do metal de transição disponível para a coordenação do monômero, obrigando-o assim à coordenação $\eta^{2}$-trans. Desse modo, a forma 1,4-trans seria obtida seguindo a rota $\mathbf{a}_{2} \rightarrow \mathbf{a}^{\prime} \rightarrow \mathbf{a}^{\mathbf{\prime}}$ (Figura 9).

\section{Terminação}

A reação de terminação pode se dar por diferentes processos, tais como: transferência com o alquilalumínio, transferência com algum composto adicionado ao meio reacional, entre outros. A transferência de cadeia com o alquilalumínio é um processo importante para os sistemas catalíticos à base de neodímio, uma vez que é o método mais prático para regular o peso molecular do polibutadieno. Nesse tipo de transferência, o sítio ativo fica disponível para iniciar uma nova cadeia polimérica.

\section{Conclusões}

Ao escolher um sistema catalítico, é importante avaliar os seus componentes, pois eles exercem influência sobre a atividade catalítica e sobre as características do polibutadieno produzido (microestrutura, peso molecular e distribuição de peso molecular). Utilizando-se catalisadores estereoespecíficos do tipo Ziegler-Natta, é possível produzir um polibutadieno com alto teor do isômero 1,4-cis. As tecnologias comercialmente disponíveis para tal produção empregam catalisadores à base de titânio, níquel, cobalto e lantanídeos, sendo estes últimos os que fornecem polímeros com alto teor de unidades 1,4-cis e, por conseguinte com melhores propriedades.

\section{Referências Bibliográficas}

1. Mano, E. B.\& Mendes L. C. - "Introdução à Polímeros", Edgard Blücher Ltda, Rio de Janeiro (1999).

2. Pires, N.M.T. - "Síntese de polibutadieno com alto teor do isômero 1,4-cis utilizando catalisadores Ziegler-Natta"; Tese de Mestrado, Instituto de Macromoléculas Professora Eloisa Mano, Universidade Federal do Rio de Janeiro (1990).

3. Pires, N.M.T - "Sistemas catalíticos à base de neodímio para obtenção polibutadieno com alto teor de unidades cis", Seminário para Doutorado, Instituto de Macromoléculas Prof. Eloisa Mano, Universidade Federal do Rio de Janeiro (2000).

4. Mello, I.L. - "Efeito do solvente na polimerização de 1,4cis de 1,3-butadieno com catalisadores à base de neodímio"; Tese de Mestrado, Instituto de Macromoléculas Professora Eloisa Mano, Universidade Federal do Rio de Janeiro (2003).

5. Mano, E. B.\& Mendes L. C. - "Introdução a Polímeros", Edgard Blücher Ltda, Rio de Janeiro, (1999).

6. Natta, G. -"Present significance of his scientific contribution", Aditrice Di Chimica, Milano, p.67-124 (1982). 
7. Boor, J. J. - “Ziegler-Natta Catalysts and polymerizations”, Academic Press, New York, p.141-145 (1979).

8. Rocha, T. C. J. - "Polibutadieno: Tipos, Características, Processos de Síntese e Aplicações", Seminário para Mestrado, Instituto de Macromoléculas Prof. Eloisa Mano, Universidade Federal do Rio de Janeiro (2003).

9. Tate, D.P. - Butadiene polymers, em "Encyclopedia of Polymer Science and Engineering" v.2, Mark, Bikales, Overberger, Menges, John Wiley \& Sons (1986).

10. Odian, G. - "Principles of polymerization",McGraw-hill Book Company, New York, 588-590(1970).

11. Fraga, L. A. - "Produção de polibutadieno alto-cis com catalisadores à base de lantanídeos", Seminário para Mestrado, Instituto de Macromoléculas Prof. Eloisa Mano, Universidade Federal do Rio de Janeiro (2000).

12. Ricci, G; Italia, S.; Cabassi, F. \& Porri L. - Polym. Commun., 28, p.223 (1987).

13. Gallazzi, C. M.; Bianchi F.; Depero, L. \& Zocchi, M. Polymer, 29, p.1516 (1988).

14. Ricci, G; Italia, S.; Comitani, C. \& Porri L. - Polym. Commun., 32 (17), p.514 (1987).

15. Jenkins, D. K. -Polymer, 33 (1), p.156 (1992).

16. Bodrova, V. S.; Piscareva, E.P.; Shelokhneva, L. F. \& Poletaeva, I. A. - Polym. Sci. Ser. A, 40 (11), p.1054 (1998).

17. Cui, L.; Ba, X.; Teng, H.; Ying, L.; Li, K. \& Yingtai, J. Polym. Bull., 40, p.729 (1998).

18. Jin, Y.; Wang, P.; Pei, F.; Cheng, G.; Cui, L. \& Song, C. Polym. Commun., 37 (2), p.349 (1996).

19. Oehme, A.; Gebauer, U. \& Gehrke, K. - Macromol. Rapid Commun. 16, p.563 (1995).

20. Ferreira, A.A. - "Estudo do efeito da cristalinidade do polibutadieno alto-cis sobre as propriedades mecânicas de suas composições com negro-de-fumo e/ou elastômero de butadieno-estireno"; Tese de Doutorado, Instituto de Macromoléculas Professora Eloisa Mano, Universidade Federal do Rio de Janeiro (2004).

21. Dolgoplosk, B.A. \& Tinyakova, E.I. -Polym. Sci. Ser. A, 36 (10), p.1380 (1994).

22. Pires, N. M. T.; Coutinho, F.M.B.; Costa, M.A.S.; Santa Maria, L.C.; Mello, I.L.; Nunes, D.S.S. - Rev. Quím. Ind. 719, p.15 (2002).

23. Oehme A.; Gebauer. U.; Gehrke, K.; Lechner M.D. - Ang. Makromol. Chem. 235, p.121 (1996).

24. Porri, L.; Giarrusso, A. and Ricci, G. - Polym. Sci., Ser. A 36 (10), p.1421 (1994).
25. Rafikov, S. R.; Monakov, Y. B.; Tolstikov, G. A.; Marina, N. G.; Minchenkova, N.K.; Savelieva, I.G. - J. Polym. Sci.: Polym. Chem., 21, p.2697 (1983).

26. Honig, J.A.J.; Burford, R.P.; Chaplin, R.P. - J. Polym. Sci.: Polym. Chem. 21, p.2559 (1983).

27. Wilson, D.J. - Makromol. Chem., Macromol. Symp. 66, p.273 (1993).

28. Zhang, Q.; Li, W.; Shen, Z. - Eur. Polym. J. 38, p.869 (2002).

29. Kobayashi, E.; Hayashi, N.; Aoshima, S.; Furukawa, J. J. Polym. Sci.: Part A: Polym. Chem. 36, p.241 (1998).

30. Marina, N.G.; Monakov, Y.B.; Rafikov, S.R.; Gadeleva, K.K. - Polym. Sci. U.S.S.R. 26 (6), p.1251 (1984).

31. Kwag, G. - Macromolecules 35 (13), p.4875 (2002).

32. Monakov, Y.B.; Marina, N.G.; Sabirov, Z.M. -Polym. Sci., Ser. A 36 (10), p.1404 (1994).

33. Rocha, T.C.J.; Soares, B.G.; Coutinho, F.M.B.; Costa, M.A.S. - Polímeros: Ciência e Tecnologia 15(1), p.39 (2005).

34. Iovu, H.; Hubca, G.; S., E.; Badea, E.; H., J. S. - Eur. Polym. J. 33 (6), p.811 (1997).

35. Pires, N.M.T. - "Estudo de sistemas catalíticos à base de neodímio para produção de polibutadieno alto-cis"; Tese de Doutorado, Instituto de Macromoléculas Professora Eloisa Mano, Universidade Federal do Rio de Janeiro (2004).

36. Rocha, T.C.J. - "Efeito de doadores de elétrons na polimerização de butadieno com catalisadores à base de neodímio"; Tese de Mestrado, Instituto de Macromoléculas Professora Eloisa Mano, Universidade Federal do Rio de Janeiro (2004).

37. Kwag, G.; Kim, P.; Han, S.; Choi, H. - Polymer 46, p.3782 (2005).

38. Kwag, G.; Lee, J.; Bae, C.; Lee, S.N. - Polymer 44, p.6555 (2003).

39. Shen, Z. - Inorg. Chim. Acta, 140, p.7 (1987).

40. Monakov, Y.B.; Sabirov, Z.M.; Marina, N.G. -Polym. Sci., Ser. A 38(3), p.232 (1996).

41. Dolgoplosk, B.A.; Tinyakova, E.I.; Yakovlev, V.A.; Gol'shtein, S.B. - Polym. Sci., Ser. A 38(3), p.261 (1996).

42. Wilson, D.J. - Polymer 34 (16), 3004 (1993).

43. Jenkins, D.K., Wilson, D.J. - Polym. Bull. 34, p.257 (1995)

44. Oehme, A.; Gebauer, U.; Gehrke, K. -J. Molec. Cat. 82, p.83 (1993). 
45. Pires, N.M.T.; Coutinho, F.M.B.; Costa, M.A.S. "Características do sistema catalítico à base de neodímio para síntese de polibutadieno alto cis", in: Anais do $5^{\circ}$ Congresso Brasileiro de Polímeros, CBPol127 (2000).

46. Koslov, V.G.; Sigalva, N.N.; Nefedjev, K.V.; Savel'eva, I.G.; Marina, N.G.; Monakov, Y.B.-- J. Polym. Sci.: Part A: Polym. Chem. 32, p.1237 (1994).

47. Nickaf, J.B., Burford, R.P., Chaplin, R.P. - J. Polym. Sci.: Part A: Polym. Chem., 33, p.1125 (1995).

48. Zhiquan, S. Jun, O., Fusong, W., Zhenya, H., Fusheng, Y., Baogong, Q. - J. Polym. Sci.: Polym. Chem. Ed., 18, p.3345 (1980)

49. Quirk, R.P.; Kells, A.M.; Yunlu, K.; Cuif, J.P. - Polymer 41, p.5903 (2000).

50. Porri, L.; Giarrusso, A.; Shubin,N.; Lu, Z.; Ricci, G. Polym. Prepr., Division of Polymer Chemistry, American Chemical Society, 39(1), p.214 (1998).

51. Wilson, D.J. - J. Polym. Sci. Part A 33, p.2505 (1995).

52. Wilson, D.J.; Jenkins, D.K. - Polym. Bull. 27, p.407 (1992).
53. Quirk, R.P.; Kells, A.M. - Polym. Int. 49 (7), p.751 (2000).

54. P. J. Ansell (Enichem Elastomers Ltd.) - "Conjugated Diene Polymerization"- Patente Americana 5.686.371, 11Nov.1997.

55. Y. C. Jang (Korea Kumho Petrochemical Co. Ltd.) "Process for preparing polybutadiene using catalyst with high activity"- Patente Americana 6.136.931, 20 Oct. 2000.

56. Nunes, D. S. S.; Coutinho, F. M. B.; Mello, I. L. \& Ferreira, A. A . - "Síntese de catalisadores à base de neodímio preparados em presença de dienos conjugados para a obtenção de polibutadieno alto-cis", in: XLIII Congresso Brasileiro Química, Ouro Preto, MG (2003).

57. Tate, D.P. - Butadiene polymers, em "Encyclopedia of Polymer Science and Engineering" v.2, Mark, Bikales, Overberger, Menges, John Wiley \& Sons (1986)

58. Stille, J.K. - "Introduction to polymer chemistry", 183186, John Wiley \& Sons, N.Y. (1967)

Enviado: 28/02/05

Reenviado: $15 / 06 / 05$

Aprovado: 28/06/05 